\title{
Inorganic Ligand-Capped Colloidal Nanocrystals for Electronic Device Application
}

\author{
Jaeyoung Jang \\ Department of Energy Engineering, Hanyang University \\ 222 Wangsimni-ro, Seongdong-gu, Seoul, South Korea \\ jyjang15@hanyang.ac.kr
}

\section{Extended Abstract}

Colloidal nanocrystals can combine the advantages of crystalline inorganic semiconductors with the size-tunable electronic structure and inexpensive solution-based device fabrication.[1] They are of great interest due to these unique advantages for use in electronic and optoelectronic devices such as field-effect transistors (FETs), photovoltaic cells, and light-emitting diodes.[1-8] Efficient charge transport is crucial for high performance of nanocrystal-based electronic and optoelectronic devices.[2,3] Many practical implementations of nanocrystals are hindered by the poor electronic coupling in close-packed nanocrystal films, caused by the presence of bulky organic surface ligands.

In this study, to address this fundamental problem, various types of inorganic surface ligands are introduced.[2,4,5] By using optimized inorganic surface ligands, nanocrystal solids are prepared exhibiting band-like charge transport, high photoconductivity and tunable doping level.[6] For example, we explore the temperature-dependent Hall effect of inorganically capped InAs nanocrystals. In addition, a solution-based "soldering" process is introduced to fabricate ultrahigh electron mobility ( $>300 \mathrm{~cm}^{2} / \mathrm{Vs}$ ) nanocrystal solids using colloidal nanocrystals with molecular "solders".[7,8] The high-mobility FETs were fabrcated by spin-coating a solution of $\mathrm{Cd}_{2} \mathrm{Se}_{3}{ }^{2-}$-capped CdSe nanocrystals, followed by thermal annealing. Finally, we expand the application of the NC soldering process to core-shell NCs consisting of a III-V InAs core and a CdSe shell with composition-matched $\mathrm{Cd}_{2} \mathrm{Se}_{3}{ }^{2-}$ molecular solders. Soldering CdSe shells forms nanoheterostructured material that combines high electron mobility and near-IR photoresponse.

\section{References}

[1] D.V. Talapin, J.-S. Lee, M.V. Kovalenko, E. V. Shevchenko, "Prospects of Colloidal Nanocrystals for Electronic and Optoelectronic Applications," Chem. Rev., vol. 110, no. 1, pp. 389-458, 2010.

[2] M. V. Kovalenko, M. Scheele, D. V. Talapin, "Colloidal Nanocrystals with Molecular Metal Chalcogenide Surface Ligands," Science, vol. 324, no. 5933, pp. 1417-1420, 2009.

[3] J.-S. Lee, M.V. Kovalenko, J. Huang, D. S. Chung, D. V. Talapin, "Band-like transport, high electron mobility and high photoconductivity in all-inorganic nanocrystal arrays," Nat. Nanotech., vol. 6, no. 6, pp. 348, 2011.

[4] A. Nag, M.V. Kovalenko, J.-S. Lee, W. Liu, B. Spokoyny, D. V. Talapin, "Metal-free Inorganic Ligands for Colloidal Nanocrystals: $\mathrm{S}^{2-}, \mathrm{HS}^{-}, \mathrm{Se}^{2-}, \mathrm{HSe}^{-}, \mathrm{Te}^{2-}, \mathrm{HTe}^{-}, \mathrm{TeS}_{3}{ }^{2-}, \mathrm{OH}^{-}$, and $\mathrm{NH}_{2}^{-}$as Surface Ligands," J. Am. Chem. Soc., vol. 133, no. 27, pp. 10612-10620, 2011.

[5] H. Zhang, J. Jang, W. Liu, D. V. Talapin, "Colloidal Nanocrystals with Inorganic Halide, Pseudohalide, and Halometallate Ligands," ACS Nano, vol. 8, no. 7, pp. 7359-7369, 2014.

[6] J. Jang, W. Liu, J. S. Son, D. V. Talapin, "Temperature-Dependent Hall and Field-Effect Mobility in Strongly Coupled All-Inorganic Nanocrystal Arrays," Nano Lett., vol. 14, no. 2, pp. 653-662, 2014.

[7] D. S. Dolzhnikov, H. Zhang, J. Jang, J. S. Son, M. G. Panthani, T. Shibata, S. Chattopadhyay, D. V. Talapin, "Composition-matched molecular "solders" for semiconductors," Science, vol. 347, no. 6220, pp. 425-428, 2015.

[8] J. Jang, D. S. Dolzhnikov, W. Liu, S. Nam, M. Shim, D. V. Talapin, "Solution-Processed Transistors Using Colloidal Nanocrystals with Composition-Matched Molecular "Solders": Approaching Single Crystal Mobility," Nano Lett., vol. 15, no. 10, pp. 6309-6317, 2015. 